\title{
Nonstandard topological extensions: Addendum
}

\section{Robert A. Herrmann}

It has recently come to my attention that, independent from my investigations in [1], Professor K.D. Stroyan [2] obtains for a Tychonoff space $X$ and a normal base $B$ a quotient space on $* X$ which is homeomorphic to the Wallman-Frink Hausdorff compactification $\omega(X, \beta)$. Consequently, if $X$ is Tychonoff, then Corollary 4.1 in [1] implies that Stroyan's space is homeomorphic to the space $\omega(X, \beta)_{S}$ which is constructed in Theorem 4.1 in [1]. Stroyan's result is obtained by use of a monadic closure operator.

I regret not mentioning Professor Stroyan's result in my paper [1].

\section{References}

[1] Robert A. Herrmann, "Nonstandard topological extensions", BuZZ. Austral. Math. Soc. 13 (1975), 269-290.

[2] K.D. Stroyan, "Additional remarks on the theory of monads", Contributions to non-standard analysis, 245-259 (Studies in Logic and the Foundations of Mathematics, 69. North-Holland, Amsterdam, London, 1972).

Department of Mathematics, United States Naval Academy, Annapolis, Maryland, USA.

Received 10 September 1975. 